\title{
Combining Traditional Accounting and Value-Added Activity Measures to Maximize Supply Chain Performance
}

\author{
Liang Xu \\ Slippery Rock University of Pennsylvania \\ L. Douglas Smith \\ University of Missouri - St. Louis
}

\begin{abstract}
When traditional accounting of revenues and costs is used in supply-chain (SC) optimizing models, there is no economic incentive to ship goods that would reach their destinations for downstream sales beyond the planning horizon. Imposing lower bounds on ending inventories is the standard remedy used to ensure that sufficient goods are on hand to support future business. It is possible, however, to refine the planning objective with a "value-added" component to provide a fuller representation of the economic value of SC activities. Planning can occur with shorter horizons. The planning process becomes more analytically tractable, and better solutions emerge.
\end{abstract}

\section{INTRODUCTION}

Mathematical optimization and discrete-event simulation can be used in concert to shape SC strategies and plan SC activities. Inferences drawn from both analytical approaches, however, can be quite sensitive to the choice of time horizon, cost-allocation methods, and the timing of recognition of value-added activities. Especially when these techniques are used with a rolling planning horizon, there are challenges of linking future plans to current operations, and revising plans as events unfold. There is a risk of making decisions which enhance performance in a particular time frame but leave the firm poorly positioned for future business.

When traditional accounting of revenues and costs is used in SC optimizing models, there is no economic incentive to ship goods that would reach their destinations for downstream sales beyond the planning horizon. Most SC optimization models (e.g., Ciarallo et al., 1994; Wang and Gerchak, 1996; Sabri and Beamon 2000; Lee and Kim, 2002; Leung et al., 2006; Lin and Chen, 2009; You et al., 2009; Cardoso et al., 2015 etc.) ignore this issue. Perhaps the authors assume implicitly that lower bounds will be placed on ending inventories to support a continuing enterprise. Such bounds would typically be set with consideration of the costs of carrying inventory (including spoilage) against the costs of failing to satisfy future (stochastic) demands.

In this paper, we employ mathematical optimization and discrete-event simulation to demonstrate how incorporating a "value-added" component in the planning objective can provide a fuller representation of the economic value of SC activities. With that enhancement, planning can occur with shorter horizons; the planning process becomes more analytically tractable; and better solutions emerge. 
Modeling in this fashion, one employs accounting with a performative rather than ostensive perspective (Boedker, 2010). The effects on performance, however, are assessed using traditional accounting methods.

\section{THE SIMULATION STUDY}

The illustrative SC (Figure 1) represents the flow of material from raw-material suppliers to manufacturing facilities where material is processed, and finally to warehouses from which product is delivered to customers. Costs of raw materials are recognized when they are shipped from the suppliers. Manufacturing costs are recognized upon completion of finished goods at the manufacturing sites. Shipping costs are recognized when goods are shipped. Carrying costs for inventories and goods in transit are recognized daily.

\section{FIGURE 1}

\section{SUPPLY CHAIN STRUCTURE}

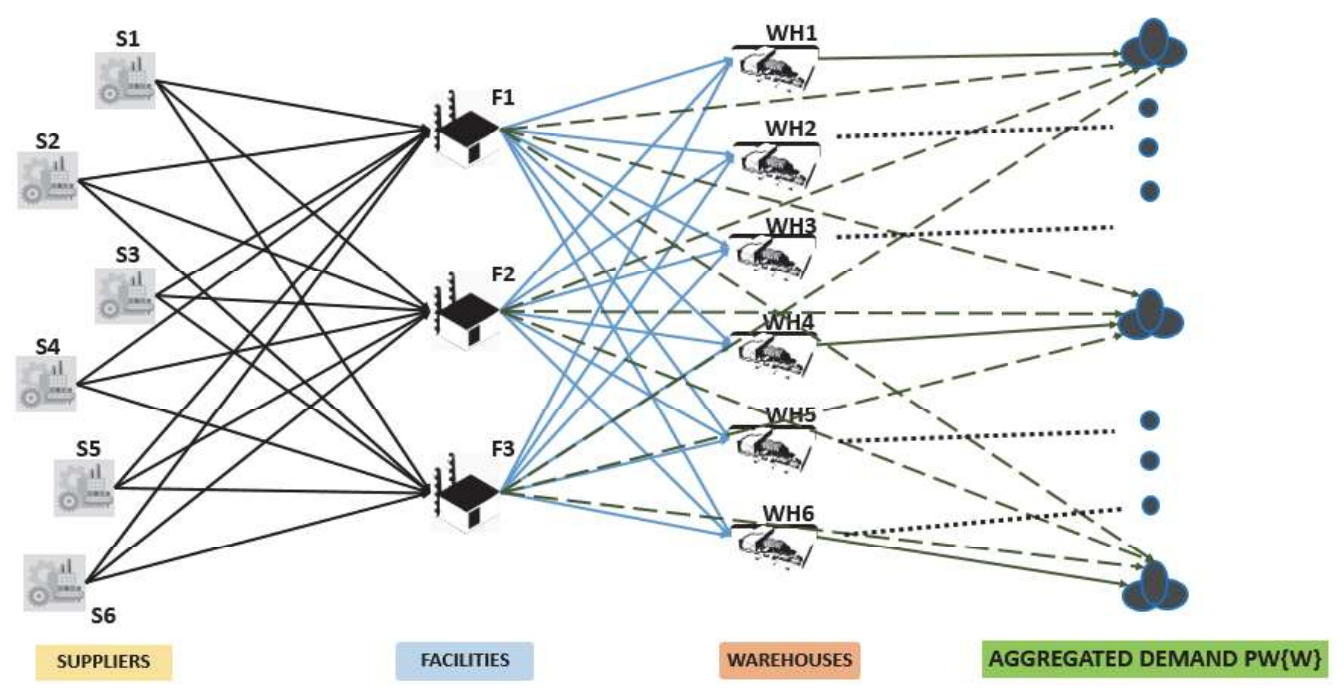

Our experimental SC has six suppliers of raw material for three production facilities that produce six bulk products which are distributed through six warehouses. Customer demands for products are aggregated daily at the individual warehouses. The locations of suppliers, production facilities, and warehouses are assumed to be fixed and transportation of raw materials and finished goods is assumed to be done by third-party logistics (3PL) providers (thus avoiding the issue of consolidating orders for distributional efficiency). The research was designed for an actual enterprise, but to preserve confidentiality, simulation parameters were chosen from other published cases (Tsiakis et al., 2001).

We utilize a discrete-event simulation model with an embedded optimizer to perform iterative updates to the plan with a rolling planning horizon over 90 days. The hybrid model is constructed on the Statistical Analysis System (SAS) 9.4 platform. This modeling approach allows us to include stochastic elements and recognize uncertainties in the SC. The analytical model is developed with the following assumptions:

1. The managerial goal is to maximize net contribution to profit as measured by traditional accounting methods.

2. Inventory replenishment occurs at the end of each business day.

3. Customer demands for products at the warehouses are registered at the beginning of each day.

4. Risk of disruptions from suppliers are independent of each other. 
5. Each production facility can produce all products, ship products to all warehouses, and perform alternative delivery of finished goods via expedited shipping (with extra costs) when outages occur at the warehouses.

Planning occurs within the framework of the SC operations reference (SCOR) model. It encompasses activities from shipment of raw material to delivery of finished goods (Bolstorff and Rosenbaum, 2007; Zhou et al., 2011). Daily production at plants, shipments to warehouses, and deliveries to customers are determined by a mixed-integer programming (MIP) model with consideration of production capacities, lower and upper inventory limits at plants and in warehouses, transit times to warehouses, and the opportunities to ship directly from production facilities to the customer (at higher cost) or accepting lost sales in the event of stockouts at the warehouses. The MIP model is provided as an appendix. With the simulation model, one can experiment with different planning horizons, intervals for updating plans, startup conditions, future demands, shipping times, etc.

Our base case involves the use of standard cash-flow accounting of costs and revenues with a penalty for lost sales. The corresponding MIP objective function is composed as follows.

Standard Objective Function:

Maximize [ Net Profit Contribution $=$ (Profit contribution from warehouse deliveries + Profit contribution from alternative deliveries - Costs of lost sales - Product inventory holding costs at plants and warehouses - Raw material inventory holding costs at plants - Product inventory shortage costs at plants and warehouses - Raw material inventory shortage costs at plants - Product inventory overstocking costs at plants and warehouses - Raw material inventory overstocking costs at plants Product shipping costs - Product in transit costs - Raw material shipping costs - Raw material in transit costs - Plant setup costs - Plant idle costs) ]

With such an objective, there is no incentive in the optimizing model to ship goods to a warehouse if the goods would not reach the warehouses in time to realize revenue from sales to customers (except to meet constraints imposed on ending inventories). To deal with the problem of truncated activity, we can add a value-added component in the objective function as follows.

We still recognize revenues from all deliveries to customers when they occur from the warehouse and when they are delivered directly to customers from the manufacturing plant. In the optimizing model, however, we additionally recognize the anticipated profit contributions from goods which will be sold beyond the planning horizon. As an approximation for this in our model, we include potential revenues for shipments that occur from production facilities to warehouses if they occur within the minimum downstream lead time plus one day of the end of the planning horizon.

Value-Added Objective Function:

Maximize [Net Profit Contribution $=$ (Profit contribution from warehouse deliveries to customers + Profit contribution from alternative deliveries + Expected profit contribution from shipments to warehouses within the minimum downstream lead time plus one day of the end of the planning horizon Costs of lost sales - Product inventory holding costs at plants and warehouses - Raw material inventory holding costs at plants - Product inventory shortage costs at plants and warehouses - Raw material inventory shortage costs at plants - Product inventory overstocking costs at plants and warehouses - Raw material inventory overstocking costs at plants - Product shipping costs - Product in transit costs - Raw material shipping costs - Raw material in transit costs - Plant setup costs - Plant idle costs) ].

To assess the impact of optimizing with one formulation versus another, we conduct 25 replications for each planning scenario with 90 days of simulated activity (an entire season) using a rolling optimization horizon. We revise schedules at the end of each simulated day to offer maximum responsiveness to immediate demands. MIP solutions for the first day's activity are used to induce production, flows of finished goods, orders from warehouses and orders of raw material in the simulation model.

The first period's product demands are presumed to be known with certainty. The simulation model generates product demands and delivery dates randomly, implements the first day of the MIP solution, and updates datasets that represent the new states of the system including finished goods in transit and raw materials in transit. Randomly generated delivery dates for raw materials and finished goods are set 
when simulated orders are placed and goods are shipped. They are not altered as successive iterations occur on the rolling horizon. The following information is used for subsequent daily revisions to the plan:

1. Raw material inventory level at each plant

2. Outstanding orders of raw materials at each plant

3. Raw materials in transit to each plant

4. Amount of each product produced at each plant

5. Finished product inventories at each plant

6. $\quad$ Finished product inventories at each warehouse

7. $\quad$ Outstanding orders of products at each warehouse

8. Finished products in transit at each warehouse.

The MIP model uses this information each simulated day as its new initial conditions and re-solves the problem for the fixed number of days in the planning horizon (e.g., Day2 to Day 16 in the second iteration of a 15-day planning horizon). This iterative process continues until we reach the end of the planning horizon (where the solution is developed for Day 90 to Day 104 and just implemented for Day 90). The optimization model and simulation models are thus used in concert to develop SC plans that attempt to maximize the net profit contribution while controlling for risk.

\section{EXPERIMENTAL RESULTS}

The SC under study has a theoretical planning horizon of at least 18 days (the expected total time required from shipment of raw material to the delivery of finished products from the warehouses. To demonstrate the effect of using the two versions of the MIP model with different planning horizons, we perform the simulation with 10-day and 20-day planning horizons and generate quarterly reports of simulated activity. Table 1 summarizes financial performance from simulations using the MIP with the standard objective function (STDOBJ) for 10-day planning horizon (H10) and 20-day planning horizon (H20). Note that shortening the planning horizon with the standard objective from 20 days to 10 days resulted in a $12 \%$ decrease in average daily profit (from $\$ 18,299$ to $\$ 16,114$ ).

TABLE 1

\section{AVERAGE DAILY NET PROFIT CONTRIBUTION WITH STDOBJ}

\begin{tabular}{|c|c|c|}
\hline \multicolumn{3}{|c|}{$\begin{array}{l}\text { Daily Net Profit Contribution Statement } \\
\text { for } 90 \text {-day period with } \mathrm{H} 10\end{array}$} \\
\hline & $\$$ & $\$$ \\
\hline GROSS PROFIT CONTRIBUTION & & $17,154.10$ \\
\hline Products sold at warehouses & $\underline{17,154.10}$ & \\
\hline PLANT EXPENSES & & $1,040.18$ \\
\hline Finished Product Inventory Costs & 9.34 & \\
\hline Finished Product in Transit Costs & 17.70 & \\
\hline Raw Material Inventory Costs & 33.11 & \\
\hline Raw Material in Transit Costs & 22.44 & \\
\hline Raw Material Shipping Costs & 449.50 & \\
\hline Idle Costs & 58.49 & \\
\hline Setup Costs & $\underline{449.60}$ & \\
\hline NET PROFIT CONTRIBUTION & & $16,113.92$ \\
\hline
\end{tabular}

\begin{tabular}{|c|c|c|}
\hline \multicolumn{3}{|c|}{$\begin{array}{l}\text { Daily Net Profit Contribution Statement } \\
\text { for } 90 \text {-day period with } \mathrm{H} 20\end{array}$} \\
\hline & $\$$ & $\$$ \\
\hline GROSS PROFIT CONTRIBUTION & & $19,269.27$ \\
\hline Products sold at warehouses & $\underline{19,269.27}$ & \\
\hline PLANT EXPENSES & & 969.89 \\
\hline Finished Product Inventory Costs & 10.26 & \\
\hline Finished Product in Transit Costs & 22.40 & \\
\hline Raw Material Inventory Costs & 39.37 & \\
\hline Raw Material in Transit Costs & 31.64 & \\
\hline Raw Material Shipping Costs & 416.22 & \\
\hline Idle Costs & 0.00 & \\
\hline Setup Costs & $\underline{450.00}$ & \\
\hline NET PROFIT CONTRIBUTION & & $18,299.38$ \\
\hline
\end{tabular}

In Table 2, we show the results when the MIP optimization occurs with the value-added component included in the objective function. Note how dramatically better the simulated performance was when the planning horizon was shorter than the total time cycle time for goods to be shipped from the warehouses. 
A little surprisingly, simulated performance with the value-added objective was slightly superior even when the planning horizon was lengthened to 20 days.

TABLE 2

AVERAGE DAILY NET PROFIT CONTRIBUTION COMPARISON WITH VAOBJ

\begin{tabular}{|lrc|}
\hline \multicolumn{2}{|c|}{$\begin{array}{c}\text { Daily Net Profit Contribution Statement for 90-day period } \\
\text { with VAOBJ and H10 }\end{array}$} & \multicolumn{1}{c|}{$\$$} \\
GROSS PROFIT CONTRIBUTION & $19,629.55$ \\
Product sold at warehouses & $19,629.55$ & \\
& & $1,045.27$ \\
PLANT EXPENSES & 10.32 & \\
Finished Product Inventory Costs & 22.93 & \\
Finished Product in Transit Costs & 38.04 & \\
Raw Material Inventory Costs & 26.00 & \\
Raw Material in Transit Costs & 497.98 & \\
Raw Material Shipping Costs & 0.00 & \\
Idle Costs & $\underline{450.00}$ & \\
Setup Costs & & \\
& & $18,584.28$ \\
\hline
\end{tabular}

\begin{tabular}{|c|c|c|}
\hline \multicolumn{3}{|c|}{$\begin{array}{l}\text { Daily Net Profit Contribution Statement for 90-day period } \\
\qquad \text { with VAOBJ and } \mathrm{H} 20\end{array}$} \\
\hline & $\$$ & $\$$ \\
\hline GROSS PROFIT CONTRIBUTION & & $19,748.32$ \\
\hline Product sold at warehouses & $19,748.32$ & \\
\hline PLANT EXPENSES & & 987.97 \\
\hline Finished Product Inventory Costs & 10.47 & \\
\hline Finished Product in Transit Costs & 24.37 & \\
\hline Raw Material Inventory Costs & 39.25 & \\
\hline Raw Material in Transit Costs & 30.82 & \\
\hline Raw Material Shipping Costs & 433.06 & \\
\hline Idle Costs & 0.00 & \\
\hline Setup Costs & $\underline{450.00}$ & \\
\hline NET PROFIT CONTRIBUTION & & $18,760.35$ \\
\hline
\end{tabular}

Finally, we test the robustness of this finding under situations where the system may be recovering from downstream disruptions. We randomly set $20 \%$ of finished-product inventories at warehouses to zero at the beginning of the planning horizon for each replication. Such situations might occur from damage in processing or shipment or following surges in demand due to interruptions in supply chains of competitors. All other initial conditions were the same as in the previous simulations. Outages could occur in any product-warehouse combination and the amounts of other inventories held in the system could be any value between min and max at beginning of each replication. The results in Table 3 show expected deterioration in average performance under each of the four scenarios but the benefits of the value-added approach persist.

TABLE 3

\section{COMPARABLE AVERAGE SC FINANCIAL PERFORMANCE WITH DISRUPTIONS}

\begin{tabular}{|c|c|c|c|c|c|c|c|c|}
\hline \multirow{3}{*}{ Scenario Two } & \multicolumn{8}{|c|}{ Daily Net Profit Contribution Statement for 90-day period } \\
\hline & \multicolumn{2}{|c|}{ STDOBJ and $\mathrm{H} 10$} & \multicolumn{2}{|c|}{ VAOBJ and $\mathrm{H} 10$} & \multicolumn{2}{|c|}{ STDOBJ and $\mathrm{H} 2 \mathrm{O}$} & \multicolumn{2}{|c|}{ VAOBJ and $\mathrm{H}_{2} \mathrm{O}$} \\
\hline & $\$$ & $\$$ & $\$$ & $\$$ & $\$$ & $\$$ & $\$$ & $\$$ \\
\hline GROSS PROFIT CONTRIBUTION & & $15,189.62$ & & $18,611.58$ & & $18,127.16$ & & $18,620.75$ \\
\hline Products sold at warehouses & $\underline{15,189.62}$ & & $18,611.58$ & & $\underline{18,127.16}$ & & $18,620.75$ & \\
\hline PLANT EXPENSES & & $1,045.40$ & & $1,045.36$ & & 971.10 & & 987.78 \\
\hline Finished Product Inventory Costs & 9.19 & & 10.33 & & 10.26 & & 10.49 & \\
\hline Finished Product in Transit Costs & 17.03 & & 23.15 & & 22.41 & & 24.53 & \\
\hline Raw Material Inventory Costs & 33.94 & & 38.00 & & 39.36 & & 39.27 & \\
\hline Raw Material in Transit Costs & 22.46 & & 26.02 & & 31.60 & & 30.79 & \\
\hline Raw Material Shipping Costs & 444.50 & & 497.86 & & 417.47 & & 432.70 & \\
\hline Idle Costs & 68.48 & & 0.00 & & 0.00 & & 0.00 & \\
\hline Setup Costs & $\underline{449.80}$ & & $\underline{450.00}$ & & $\underline{450.00}$ & & $\underline{450.00}$ & \\
\hline NET PROFIT CONTRIBUTION & & $14,144.22$ & & $17,566.22$ & & $17,156.06$ & & $17,632.97$ \\
\hline
\end{tabular}


Further insights about SC performance can be obtained from statistical analysis of simulated performance at the product level. For that analysis, we produced the following performance metrics:

- Average daily gross profit contribution

- Average daily product deliveries from warehouses

- Average daily product shipments to customers from plants

- Average daily lost sales for each product

- Percentages of product demands satisfied from warehouse deliveries

- Average supply chain service level for individual products

- Average daily production of each product (across all plants)

- Average daily warehouses warehouse inventory in days of demands

- Average ending inventory (in days of demand for each product) upon completion of simulated activity

- Average total plant finished inventory (in days of total demand for each product)

- Average total plant end finished product inventory in days of demands.

Ending inventories are important to consider because they position the firm for ongoing operations beyond the simulated period. Statistics for overall SC performance on all eleven metrics are presented at the product level in Table 4 for scenarios with the downstream supply disruptions.

Table 4 reveals how the value-added complement to the optimization model's objective function improves products' daily gross profit contribution, increases warehouse deliveries to satisfy customer demands, reduces alternative (more expensive) deliveries and lost sales, and improves warehouses' product service level. More products are being produced across production facilities. For the same length of planning horizon ( $\mathrm{H} 10$ or $\mathrm{H} 20)$, more buffer inventories were held in the system when value-added complement to the objective function was used. Statistical analysis of the findings using ANOVA and pairwise comparisons with Duncan's multiple range tests on all eleven metrics (not shown here) affirm that our findings demonstrate systematic rather than random benefits in employing the value-added complement in the MIP formulation. 
TABLE 4

PRODUCT-LEVEL PERFORMANCE STATISTICS WITH DISRUPTIONS

\begin{tabular}{|c|c|c|c|c|c|c|c|c|c|c|c|c|c|}
\hline Product & $\begin{array}{c}\text { Expected } \\
\text { Daily } \\
\text { Demand }\end{array}$ & $\begin{array}{l}\text { Avg. } \\
\text { Simul. } \\
\text { Daily } \\
\text { Demand }\end{array}$ & $\begin{array}{l}\text { Avg. } \\
\text { Daily } \\
\text { Shipped } \\
\text { From } \\
\text { Whses } \\
\end{array}$ & $\begin{array}{l}\text { Avg. } \\
\text { Daily } \\
\text { Shipped } \\
\text { From } \\
\text { Plants } \\
\end{array}$ & $\begin{array}{l}\text { Avg. } \\
\text { Daily } \\
\text { Lost } \\
\text { Sales }\end{array}$ & $\begin{array}{l}\text { Avg. } \\
\text { Daily } \\
\text { Gross } \\
\text { Profit } \\
\text { Contrib } \\
\end{array}$ & $\begin{array}{c}\text { Avg. } \\
\text { Daliy } \\
\text { Whse Deliv } \\
\text { (pet) }\end{array}$ & $\begin{array}{l}\text { Avg. SC } \\
\text { Service } \\
\text { Level } \\
\text { (pct) }\end{array}$ & $\begin{array}{l}\text { Avg. } \\
\text { Dally } \\
\text { Total Plant } \\
\text { Produced } \\
\end{array}$ & $\begin{array}{l}\text { Avg. } \\
\text { Total } \\
\text { Whse Inv } \\
\text { (days } \\
\text { demand) }\end{array}$ & $\begin{array}{l}\text { Avg. } \\
\text { Total Whse } \\
\text { End Inv } \\
\text { (days } \\
\text { demand) }\end{array}$ & $\begin{array}{l}\text { Avg. } \\
\text { Totai } \\
\text { Plant Inv } \\
\text { (days } \\
\text { demand) }\end{array}$ & $\begin{array}{l}\text { Avg. } \\
\text { Total Plant } \\
\text { End Inv } \\
\text { (days } \\
\text { demand) }\end{array}$ \\
\hline 1 & 7020 & 698.8 & 508.5 & 108.7 & 815 & 3,616 & 728 & 88.4 & 595.3 & 1.8 & 1.0 & 0.8 & 0.8 \\
\hline 2 & 984.0 & 980.0 & 742.2 & 138.7 & 99.1 & 4,865 & 75.7 & 899 & 8459 & 20 & 1.0 & 0.9 & 0.8 \\
\hline 3 & 273.0 & 269.7 & 1923 & 45.6 & 318 & 1,730 & 713 & 88.4 & 228.7 & 22 & 1.2 & 0.8 & 0.9 \\
\hline 4 & 381.0 & 378.1 & 268.2 & 63.2 & 467 & 2,178 & 70.9 & 878 & 319.9 & 1.7 & 1.1 & 0.8 & 0.8 \\
\hline 5 & 780 & 75.7 & 55.0 & 13.0 & 7.8 & 1,286 & 72.7 & 89.8 & 65.3 & 3.0 & 2.1 & 0.9 & 0.8 \\
\hline \multirow[t]{2}{*}{6} & 105.0 & 1027 & 71.2 & 19.2 & 123 & 1.515 & 69.4 & 88.0 & 86.6 & 2.3 & 1.3 & 0.8 & 0.8 \\
\hline & $2,523.0$ & $2,505.1$ & $1,837.4$ & 388.4 & 279.3 & 15,190 & & & $2,141.7$ & & & & \\
\hline
\end{tabular}

Summary Statistics for Quarterly Product-level Supply-Chain Metrics in 25 Replications Scenario Two 10-Day Planning Horizon and VA_Objective

\begin{tabular}{|c|c|c|c|c|c|c|c|c|c|c|c|c|c|}
\hline Product & $\begin{array}{c}\text { Expected } \\
\text { Dally } \\
\text { Demand }\end{array}$ & $\begin{array}{l}\text { Avg. } \\
\text { Simul. } \\
\text { Daily } \\
\text { Demand }\end{array}$ & $\begin{array}{l}\text { Avg. } \\
\text { Daily } \\
\text { Shipped } \\
\text { From } \\
\text { Whses } \\
\end{array}$ & $\begin{array}{l}\text { Avg. } \\
\text { Daily } \\
\text { shipped } \\
\text { From } \\
\text { Plants } \\
\end{array}$ & $\begin{array}{l}\text { Avg. } \\
\text { Daily } \\
\text { Lost } \\
\text { Sales }\end{array}$ & $\begin{array}{l}\text { Avg. } \\
\text { Daily } \\
\text { Gross } \\
\text { Profit } \\
\text { Contrib } \\
\end{array}$ & $\begin{array}{c}\text { Avg. } \\
\text { Daily } \\
\text { Whise Deliv } \\
\text { (pct) }\end{array}$ & $\begin{array}{l}\text { Avg. SC } \\
\text { Service } \\
\text { Level } \\
\text { (pct) }\end{array}$ & $\begin{array}{l}\text { Avg. } \\
\text { Daily } \\
\text { Total Plant } \\
\text { Produced } \\
\end{array}$ & $\begin{array}{l}\text { Avg. } \\
\text { Total } \\
\text { Whse inv } \\
\text { (days } \\
\text { demand) }\end{array}$ & $\begin{array}{l}\text { Avg. } \\
\text { Total Whse } \\
\text { End inv } \\
\text { (days } \\
\text { demand) }\end{array}$ & $\begin{array}{l}\text { Avg. } \\
\text { Total } \\
\text { Plant Inv } \\
\text { (days } \\
\text { demand) }\end{array}$ & $\begin{array}{l}\text { Avg. } \\
\text { Total Plant } \\
\text { End Inv } \\
\text { (days } \\
\text { demand) }\end{array}$ \\
\hline 1 & 702.0 & 698.8 & 645.0 & 19.1 & 34.6 & 4,679 & 92.3 & 95.1 & 684.1 & 4.7 & 52 & 1.0 & 1.0 \\
\hline 2 & 984.0 & 980.0 & 913.2 & 23.3 & 43.5 & 6,002 & 93.2 & 95.6 & 962.1 & 4.9 & 53 & 1.0 & 1.0 \\
\hline 3 & 273.0 & 2697 & 181.9 & 48.3 & 39.5 & 1.563 & 67.5 & 85.4 & 2220 & 24 & 1.8 & 0.8 & 0.8 \\
\hline 4 & 381.0 & 378.1 & 3072 & 39.7 & 312 & 2,573 & 812 & 91.7 & 342.2 & 2.3 & 20 & 0.9 & 0.9 \\
\hline 5 & 78.0 & 75.7 & 73.1 & 0.5 & 2.2 & 1.685 & 96,5 & 972 & 77.8 & 6.6 & 74 & 10 & 1.0 \\
\hline \multirow[t]{2}{*}{6} & 105.0 & 102.7 & 98.6 & 0.4 & 3.7 & 2,111 & 96.0 & 96.4 & 1062 & 6.2 & 72 & 1.0 & 1.0 \\
\hline & 2.523 .0 & $2,505.1$ & 2.219 .0 & 131.3 & 154.7 & 18.612 & & & 2.394 .4 & & & & \\
\hline
\end{tabular}

Summary Statistics for Quarterly Product-level Supply-Chain Metrics in 25 Replications Scenario Two 20-Day Planning Horizon and STD Objective

\begin{tabular}{|c|c|c|c|c|c|c|c|c|c|c|c|c|c|}
\hline Product & $\begin{array}{l}\text { Expected } \\
\text { Daily } \\
\text { Demand }\end{array}$ & $\begin{array}{l}\text { Avg. } \\
\text { Simul. } \\
\text { Daily } \\
\text { Demand }\end{array}$ & $\begin{array}{l}\text { Avg. } \\
\text { Daily } \\
\text { Shipped } \\
\text { From } \\
\text { Whses }\end{array}$ & $\begin{array}{l}\text { Avg. } \\
\text { Daily } \\
\text { Shipped } \\
\text { From } \\
\text { Plants }\end{array}$ & $\begin{array}{l}\text { Avg. } \\
\text { Daily } \\
\text { Lost } \\
\text { Sales }\end{array}$ & $\begin{array}{l}\text { Avg. } \\
\text { Daily } \\
\text { Gross } \\
\text { Profit } \\
\text { Contrib }\end{array}$ & $\begin{array}{l}\text { Avg. } \\
\text { Daily } \\
\text { Whse Deilv } \\
\text { (pet) }\end{array}$ & $\begin{array}{l}\text { Avg. Sc } \\
\text { Service } \\
\text { Level } \\
\text { (pet) }\end{array}$ & $\begin{array}{l}\text { Avg. } \\
\text { Daliy } \\
\text { Total Plant } \\
\text { Produced }\end{array}$ & $\begin{array}{l}\text { Avg. } \\
\text { Total } \\
\text { Whse inv } \\
\text { (days } \\
\text { demand) }\end{array}$ & $\begin{array}{l}\text { Avg. } \\
\text { Total Whse } \\
\text { End inv } \\
\text { (days } \\
\text { demand) }\end{array}$ & $\begin{array}{l}\text { Avg. } \\
\text { Total } \\
\text { Plant inv } \\
\text { (days } \\
\text { demand) }\end{array}$ & $\begin{array}{l}\text { Avg. } \\
\text { Total Plant } \\
\text { End inv } \\
\text { (days } \\
\text { demand) }\end{array}$ \\
\hline 1 & 702.0 & 698.8 & 635.9 & 25.6 & 37.3 & 4,583 & 910 & 94.7 & 677.4 & 4.7 & 50 & 1.0 & 1.0 \\
\hline 2 & 9840 & 9600 & 905.5 & 279 & 457 & 5.914 & 925 & 953 & 957.4 & 4.9 & 5.1 & 1.0 & 1.0 \\
\hline 3 & 273.0 & 2697 & 165.7 & 562 & 479 & 1,320 & 61.4 & 82.3 & 219.5 & 3.6 & 3.1 & 0.8 & 0.8 \\
\hline 4 & 3810 & 378.1 & 306.4 & 41.1 & 30.5 & 2,566 & 81.0 & 919 & 341.3 & 23 & 18 & 09 & 0.9 \\
\hline 5 & 780 & 75.7 & 726 & 0.6 & 25 & 1,656 & 95.9 & 96.7 & 77.5 & 72 & 78 & 1.0 & 1.0 \\
\hline \multirow[t]{2}{*}{6} & 105.0 & 102.7 & 99.0 & 0.4 & 3.3 & 2,088 & 96.4 & 96.8 & 105.0 & 6.7 & 7.7 & 10 & 1.0 \\
\hline & $2,523.0$ & $2,505.1$ & $2,186.1$ & 151.8 & 167.2 & 18,127 & & & $2,378.2$ & & & & \\
\hline
\end{tabular}

Summary Statistics for Quarterly Product-level Supply-Chain Metrics in 25 Replications Scenario Two 20-Day Planning Horizon and VA_Objective

\begin{tabular}{|c|c|c|c|c|c|c|c|c|c|c|c|c|c|}
\hline Product & $\begin{array}{c}\text { Expected } \\
\text { Dolly } \\
\text { Demand }\end{array}$ & $\begin{array}{l}\text { Avg. } \\
\text { Simul. } \\
\text { Daily } \\
\text { Demand }\end{array}$ & $\begin{array}{l}\text { Avg. } \\
\text { Daily } \\
\text { Shipped } \\
\text { From } \\
\text { Whses }\end{array}$ & $\begin{array}{l}\text { Avg. } \\
\text { Daify } \\
\text { Shipped } \\
\text { From } \\
\text { Plants }\end{array}$ & $\begin{array}{l}\text { Avg. } \\
\text { Daily } \\
\text { Lost } \\
\text { Sales }\end{array}$ & $\begin{array}{l}\text { Avg: } \\
\text { Daily } \\
\text { Gross } \\
\text { Profit } \\
\text { Contrib }\end{array}$ & $\begin{array}{c}\text { Avg. } \\
\text { Daily } \\
\text { Whse Delliv } \\
\text { (pct) }\end{array}$ & $\begin{array}{l}\text { Avg. Sc } \\
\text { Service } \\
\text { Level } \\
\text { (pct) }\end{array}$ & $\begin{array}{l}\text { Avg. } \\
\text { Daily } \\
\text { Totat Plant } \\
\text { Produced }\end{array}$ & $\begin{array}{l}\text { Avg. } \\
\text { Total } \\
\text { Whse Inv } \\
\text { (days } \\
\text { demand) }\end{array}$ & $\begin{array}{l}\text { Avg. } \\
\text { Total Whse } \\
\text { End Inv } \\
\text { (days } \\
\text { demand) }\end{array}$ & $\begin{array}{l}\text { Avg. } \\
\text { Total } \\
\text { Plant Inv } \\
\text { (days } \\
\text { demand) }\end{array}$ & $\begin{array}{l}\text { Avg. } \\
\text { Total Piant } \\
\text { End inv } \\
\text { (days } \\
\text { demand) }\end{array}$ \\
\hline 1 & 7020 & 698.8 & 676.6 & 0.0 & 22.2 & 4,957 & 96.8 & 96.8 & 702.0 & 5.1 & 5.7 & 10 & 1.0 \\
\hline 2 & 984.0 & 9800 & 952.2 & 0.0 & 278 & 6,331 & 972 & 972 & 985.2 & 5.0 & 5.4 & 1.0 & 1.0 \\
\hline 3 & 273.0 & 2697 & 180.5 & 0.0 & 892 & 535 & 669 & 669 & 176.0 & 3.5 & 3.1 & 0.6 & 0.7 \\
\hline 4 & 381.0 & 378.1 & 360.3 & 0.0 & 17.8 & 2,994 & 953 & 95.3 & 389.0 & 5.0 & 5.1 & 10 & 1.0 \\
\hline 5 & 780 & 757 & 73.4 & 0.0 & 23 & 1,688 & 969 & 96.9 & 78.8 & 70 & 7.7 & 1.0 & 1.0 \\
\hline \multirow[t]{2}{*}{6} & 105,0 & 1027 & 99.7 & 0.0 & 30 & 2,116 & 97.1 & 97.1 & 105.7 & 6.5 & 73 & 1.0 & 1.0 \\
\hline & $2,523.0$ & 2.505 .1 & 2.342 .7 & 0.0 & 162.3 & 18,621 & & & 2.436 .8 & & & & \\
\hline
\end{tabular}




\section{CONCLUSION AND FUTURE RESEARCH}

It is generally recognized that choice of accounting standards (Sepehri and Houmes, 2011; Sedki et al., 2014), capital structure (Cole et al., 2015), and inventory strategies (Frankel \& Hsu, 2015) all have significant impacts on corporate financial performance. Similarly, solutions from models designed to optimize supply chains can be quite sensitive to the ways that revenues and costs are recognized. In this research, we have demonstrated how SC performance can be sensitive to the length of the planning horizon when standard cash-flow accounting of costs and revenues is employed in a SC optimizing model. Use of an optimizing model can be counterproductive if planning occurs with too short a planning horizon. Our alternative formulation with a value-added complement proved to be an effective antidote which enabled MIP optimization to be employed with shorter planning horizons. The advantages of our alternative formulation persisted when downstream inventory outages were imposed randomly at the beginning of the planning horizon.

In future research, the SC under investigation will be stressed by incorporating additional disruptions upstream and at plants. With our blend of simulation and optimization, we shall test the effectiveness of combining routine $\mathrm{SC}$ risk reduction strategies with strategies for managing adverse events.

\section{REFERENCES}

Aliabadi, S., Dorestani, A., \& Balsara, N. (2013). The Most Value Relevant Accounting Performance Measure by Industry. Journal of Accounting \& Finance (2158-3625), 13(1), 22-34.

Boedker, C. (2010). Ostensive versus performative approaches for theorising accounting-strategy research. Accounting, Auditing \& Accountability Journal; Bradford, 23(5), 595-625.

Bolstorff, P., \& Rosenbaum, R. (2007). Supply Chain Excellence: A Handbook for Dramatic Improvement Using the SCOR Model (Vol. 2nd ed). New York: AMACOM.

Cardoso, S. R., Paula Barbosa-Póvoa, A., Relvas, S., \& Novais, A. Q. (2015). Resilience metrics in the assessment of complex supply-chains performance operating under demand uncertainty. Omega, 56, 53-73.

Ciarallo, F. W., Akella, R., \& Morton, T. E. (1994). A Periodic Review, Production Planning Model with Uncertain Capacity and Uncertain Demand-Optimality of Extended Myopic Policies. Management Science, 40(3), 320-332.

Corey Cole, Ying Yan, \& David Hemley. (2015). Does Capital Structure Impact Firm Performance: An Empirical Study of Three U.S. Sectors. Journal of Accounting \& Finance (2158-3625), 15(6), $57-65$.

Frankel, M., \& Hsu, P.-H. (2015). LIFO Adoption by Industry. Journal of Accounting \& Finance (2158$3625), 15(5), 42-53$.

Lee, Y. H., \& Kim, S. H. (2002). Production-distribution planning in supply chain considering capacity constraints. Computers \& Industrial Engineering, 43(1-2), 169-190.

Leung, S. C. H., Wu, Y., \& Lai, K. K. (2006). A Stochastic Programming Approach for Multi-Site Aggregate Production Planning. The Journal of the Operational Research Society, 57(2), 123132.

Lin, C.-T., \& Chen, Y. M. (2009). Hedging strategic flexibility in the distribution optimization problem. Omega, 37(4), 826-837.

Sabri, E. H., \& Beamon, B. M. (2000). A multi-objective approach to simultaneous strategic and operational planning in supply chain design. Omega, 28(5), 581-598.

Sam Sedki, S., Smith, A., \& Strickland, A. (2014). Differences and Similarities Between IFRS and GAAP on Inventory, Revenue Recognition and Consolidated Financial Statements. Journal of Accounting \& Finance (2158-3625), 14(2), 120-123.

Sepehri, M., \& Houmes, R. (2011). A Comparative Review and Analysis of the Movement Toward a Global Accounting Standard. Journal of Accounting \& Finance (2158-3625), 11(3), 53-61. 
Tsiakis, P., Shah, N., \& Pantelides, C. C. (2001). Design of Multi-echelon Supply Chain Networks under Demand Uncertainty. Industrial \& Engineering Chemistry Research, 40(16), 3585-3604.

Wang, Y., \& Gerchak, Y. (1996). Periodic Review Production Models with Variable Capacity, Random Yield, and Uncertain Demand. Management Science, 42(1), 130-137.

You, F., Wassick, J. M., \& Grossmann, I. E. (2009). Risk management for a global supply chain planning under uncertainty: Models and algorithms. AIChE Journal, 55(4), 931-946.

Zhou, H., Benton, W. C., Schilling, D. A., \& Milligan, G. W. (2011). Supply Chain Integration and the SCOR Model. Journal of Business Logistics, 32(4), 332-344.

\section{APPENDIX}

Set notation employed

\begin{tabular}{ll}
\hline Set & Description \\
$\mathrm{R}\{\mathrm{r}\}$ & Set of raw materials \\
$\mathrm{S}\{\mathrm{s}\}$ & Set of suppliers \\
$\mathrm{F}\{\mathrm{f}\}$ & Set of production facilities \\
$\mathrm{P}\{\mathrm{p}\}$ & Set of products \\
$\mathrm{W}\{\mathrm{w}\}$ & Set of warehouses \\
$\mathrm{D}\{\mathrm{d}\}$ & Set of days in planning horizon \\
$\mathrm{SR}\{\mathrm{r}\}$ & Set of suppliers for raw material $\mathrm{r}$ \\
$\mathrm{RP}\{\mathrm{p}\}$ & Set of raw materials used in producing product $\mathrm{p}$ \\
$\mathrm{PF}\{\mathrm{f}\}$ & Set of products produced in production facility $\mathrm{f}$ \\
$\mathrm{PR}\{\mathrm{r}\}$ & Set of products require raw material $\mathrm{r}$ for production \\
$\mathrm{RF}\{\mathrm{f}\}$ & Set of raw materials used in producing products at production facility $\mathrm{f}$ \\
$\mathrm{PW}\{\mathrm{w}\}$ & Set of products distributed through warehouse $\mathrm{w}$ \\
$\mathrm{WP}\{\mathrm{p}\}$ & Set of warehouses to which product $\mathrm{p}$ is delivered \\
DRMS $\{\mathrm{r}, \mathrm{s}, \mathrm{f}\}$ & Set of days raw material $\mathrm{r}$ from supplier s to arrive at production facility $\mathrm{f}$ \\
$\mathrm{DFGS}\{\mathrm{p}, \mathrm{f}, \mathrm{w}\}$ & Set of days product $\mathrm{p}$ from production facility $\mathrm{f}$ to arrive at warehouse $\mathrm{w}$ \\
\hline
\end{tabular}

Optimization Model Parameters

\begin{tabular}{|c|c|}
\hline Parameter & Description \\
\hline $\mathrm{mrR}_{\mathrm{r}} \mathrm{P}_{\mathrm{p}}$ & Units of raw material $r$ required to produce one unit of product $p$ \\
\hline $\operatorname{mininv} \mathrm{P}_{\mathrm{p}} \mathrm{F}_{\mathrm{f}}$ & Minimum inventory of product $\mathrm{p}$ desired at production facility $\mathrm{f}$ \\
\hline $\operatorname{maxinv} \mathrm{P}_{\mathrm{p}} \mathrm{F}_{\mathrm{f}}$ & Maximum inventory of product $p$ desired at production facility $f$ \\
\hline $\operatorname{mininv} R_{r} F_{f}$ & Minimum inventory of raw material $r$ desired at production facility $f$ \\
\hline $\operatorname{maxinv} R_{\mathrm{r}} \mathrm{F}_{\mathrm{f}}$ & Maximum inventory of raw material $r$ desired at production facility $f$ \\
\hline ShtPenalty $\mathrm{R}_{\mathrm{r}} \mathrm{F}_{\mathrm{f}}$ & Inventory shortage cost/unit/day of raw material $r$ at production facility $f$ \\
\hline ShtPenalty $\mathrm{P}_{\mathrm{p}} \mathrm{F}_{\mathrm{f}}$ & Inventory shortage cost/unit/day of product $\mathrm{p}$ at production facility $\mathrm{f}$ \\
\hline ShtPenalty $\mathrm{P}_{\mathrm{p}} \mathrm{W}_{\mathrm{w}}$ & Inventory shortage cost/unit/day of product $\mathrm{p}$ at warehouse $\mathrm{w}$ \\
\hline OvrPenalty $\mathrm{R}_{\mathrm{r}} \mathrm{F}_{\mathrm{f}}$ & Inventory overage cost/unit/day of raw material $\mathrm{r}$ at production facility $\mathrm{f}$ \\
\hline OvrPenalty $\mathrm{P}_{\mathrm{p}} \mathrm{F}_{\mathrm{f}}$ & Inventory overage cost/unit/day of product $p$ at production facility $f$ \\
\hline OvrPenaltyP $\mathrm{P}_{\mathrm{p}} \mathrm{W}_{\mathrm{w}}$ & Inventory overage cost/unit/day of product $\mathrm{p}$ at warehouse $\mathrm{w}$ \\
\hline $\operatorname{mininv} \mathrm{P}_{\mathrm{p}} \mathrm{W}_{\mathrm{w}}$ & Min inventory of product $\mathrm{p}$ at warehouse w (including outstanding orders) \\
\hline $\operatorname{maxinv} \mathrm{P}_{\mathrm{p}} \mathrm{W}_{\mathrm{w}}$ & Max inventory of product $\mathrm{p}$ at warehouse $\mathrm{w}$ (including outstanding orders) \\
\hline
\end{tabular}




\begin{tabular}{|c|c|}
\hline dem $_{\mathrm{p}} \mathrm{whse}_{\mathrm{w}}$ & Assigned aggregated average daily demand for product $\mathrm{p}$ at warehouse $\mathrm{w}$ \\
\hline shiptimeF $_{\mathrm{f}} \mathrm{W}_{\mathrm{w}}$ & Shipping time (days) from production facility $\mathrm{f}$ to warehouse $\mathrm{w}$ \\
\hline shiptime $\mathrm{S}_{\mathrm{s}} \mathrm{F}_{\mathrm{f}}$ & Shipping time (days) from supplier $s$ to production facility $f$ \\
\hline $\mathrm{spcF}_{\mathrm{f}}$ & Setup costs at production facility f each day that production occurs \\
\hline $\mathrm{pcP}_{\mathrm{p}} \mathrm{W}_{\mathrm{w}}$ & Unit profit contribution of product $\mathrm{p}$ delivered from warehouse $\mathrm{w}$ \\
\hline $\mathrm{scP}_{\mathrm{p}} \mathrm{F}_{\mathrm{f}} \mathrm{W}_{\mathrm{w}}$ & Cost per unit of product $\mathrm{p}$ from production facility $\mathrm{f}$ to warehouse $\mathrm{w}$ \\
\hline $\mathrm{scR}_{\mathrm{r}} \mathrm{S}_{\mathrm{S}} \mathrm{F}_{\mathrm{f}}$ & Cost per unit of raw material $r$ from supplier $s$ to production facility $f$ \\
\hline $\mathrm{scP}_{\mathrm{p}} \mathrm{W}_{\mathrm{w}}$ & Shipping cost per unit of product $\mathrm{p}$ from warehouse $\mathrm{w}$ to customer \\
\hline $\mathrm{icP}_{\mathrm{p}} \mathrm{F}_{\mathrm{f}}$ & Inventory carrying cost for finished product $\mathrm{p}$ at production facility $\mathrm{f}$ \\
\hline $\mathrm{icR}_{\mathrm{r}} \mathrm{F}_{\mathrm{f}}$ & Inventory carrying cost of raw material $r$ at production facility $f$ \\
\hline $\operatorname{itcP}_{\mathrm{p}} \mathrm{F}_{\mathrm{f}} \mathrm{W}_{\mathrm{w}}$ & In transit cost/unit of product $\mathrm{p}$ from production facility $\mathrm{f}$ to warehouse $\mathrm{w}$ \\
\hline $\operatorname{itcR}_{\mathrm{r}} \mathrm{S}_{\mathrm{s}} \mathrm{F}_{\mathrm{f}}$ & In transit cost/unit of raw material $r$ from supplier $s$ to production facility $f$ \\
\hline $\mathrm{acP}_{\mathrm{p}} \mathrm{F}_{\mathrm{f}} \mathrm{W}_{\mathrm{w}}$ & Alt. supply cost/unit of $\mathrm{p}$ from production facility $\mathrm{f}$ to warehouse $\mathrm{w}$ \\
\hline $\operatorname{icP}_{\mathrm{p}} \mathrm{W}_{\mathrm{w}}$ & Inventory carrying cost for product $\mathrm{p}$ at warehouse $\mathrm{w}$ \\
\hline $\operatorname{opcost}_{\mathrm{p}} \mathrm{W}_{\mathrm{w}}$ & Unit opportunity cost of lost sales for product $\mathrm{p}$ at warehouse $\mathrm{w}$ \\
\hline $\operatorname{DemP}_{\mathrm{p}} \mathrm{W}_{\mathrm{w}} \mathrm{D}_{\mathrm{d}}$ & Demand for product $\mathrm{p}$ (units) at warehouse $\mathrm{w}$ on day $\mathrm{d}$ \\
\hline sutime $P_{p} F_{f}$ & Product $\mathrm{p}$ production setup time at production facility $\mathrm{f}$ \\
\hline idlePenF $_{f}$ & Idle penalty cost per hour at production facility $\mathrm{f}$ \\
\hline MxprodF $_{f}$ & Maximum daily throughput (units) at production facility $f$ \\
\hline minsysinv $P_{p}$ & Desired min system inventory of product $\mathrm{p}$ (across all production facilities) \\
\hline $\operatorname{maxsy\operatorname {sinv}} \mathrm{P}_{\mathrm{p}}$ & Desired max system inventory of product $\mathrm{p}$ (across all production facilities) \\
\hline
\end{tabular}

The algebraic formulation of the problem is presented below:

$$
\begin{aligned}
& \operatorname{Max} \sum_{d \in D\{d\}}\left\{\sum _ { w \in W \{ w \} } \sum _ { p \in P W \{ w \} } \left[\left(p c P_{p} W_{w}-s c P_{p} W_{w}\right) * \operatorname{DelP}_{p} W_{w} D_{d}\right.\right. \\
& +\sum_{f \in F\{f\}}\left(p c P_{p} W_{w}-a c P_{p} F_{f} W_{w}\right) * A l t P_{p} F_{f} W_{w} D_{d}-o p \cos t P_{p} W_{w} * L S P_{p} W_{w} D_{d}-i c P_{p} W_{w} \\
& \text { *Inv } \left.P_{p} W_{w} D_{d}-\operatorname{ShtPenalty} P_{p} W_{w} * U S P_{p} W_{w} D_{d}-\text { OvrPenalty } P_{p} W_{w} * O S P_{p} W_{w} D_{d}\right] \\
& -\sum_{f \in F\{f\}}\left[\sum _ { p \in P \{ p \} } \left(i c P_{p} F_{f} * I n v P_{p} F_{f} D_{d}+\operatorname{ShtPenalty} P_{p} F_{f} * U S P_{p} F_{f} D_{d}+\text { OvrPenalty } P_{p} F_{f}\right.\right. \\
& \left.* O S P_{p} F_{f} D_{d}+\sum_{w \in W\{w\}}\left(s c P_{p} F_{f} W_{w} * S h p P_{p} F_{f} W_{w} D_{d}+i t c P_{p} F_{f} W_{w} * \operatorname{Tr} P_{p} F_{f} W_{w} D_{d}\right)\right)+s p c F_{f} \\
& \text { *SUF } F_{f}+i d l e P e n F_{f} * i d l e F_{f} D_{d} \\
& +\sum_{r \in R P\{p\}}\left(i c R_{r} F_{f} * I n v R_{r} F_{f} D_{d}+\operatorname{ShtPenalty} R_{r} F_{f} * U S R_{r} F_{f} D_{d}+\text { OvrPenalty } R_{r} F_{f}\right. \\
& \text { * OSR } \left.\left.\left.R_{r} F_{f} D_{d}+\sum_{s \in S R\{r\}}\left(s c R_{r} S_{s} F_{f} * \operatorname{Shp} R_{r} S_{s} F_{f} D_{d}+i t c R_{r} S_{s} F_{f} * \operatorname{Tr} R_{r} S_{s} F_{f} D_{d}\right)\right)\right]\right\}
\end{aligned}
$$




\section{Subject to the following constraints:}

Product $\mathrm{p}$ can't be produced at production facility $\mathrm{f}$ on day $\mathrm{d}$ unless the necessary set up is completed (constraint $\mathrm{STP}_{\mathrm{p}} \mathrm{F}_{\mathrm{f}} \mathrm{D}_{\mathrm{d}}$ ). For each production facility and day for each $p \in P F\{f\}$,

$\operatorname{ProdP}_{\mathrm{p}} \mathrm{F}_{\mathrm{f}} \mathrm{D}_{\mathrm{d}} \leq \operatorname{MxprodP}_{\mathrm{p}} \mathrm{F}_{\mathrm{f}} * \operatorname{SUP}_{\mathrm{p}} \mathrm{F}_{\mathrm{f}} \mathrm{D}_{\mathrm{d}}$.

Consumption of raw materials $\mathrm{r}$ at production facility $\mathrm{f}$ on day $\mathrm{d}$ cannot exceed the quantities available at beginning of day $d$ (constraint $U_{B R} F_{\mathrm{r}} \mathrm{D}_{\mathrm{d}}$ ). For each production facility and day for each $p \in P R\{r\}$ and each $r \in R F\{f\}$,

$\sum_{p \in P R\{r\}} \operatorname{mrR}_{\mathrm{r}} \mathrm{P}_{\mathrm{p}} * \operatorname{ProdP}_{\mathrm{p}} \mathrm{F}_{\mathrm{f}} \mathrm{D}_{\mathrm{d}} \leq \operatorname{InvR}_{\mathrm{r}} \mathrm{F}_{\mathrm{f}} \mathrm{D}_{\mathrm{d}}$

Notice that if units of raw material $r$ required to produce each unit of product $p$ are significantly different across production facilities because of labor, technology, or machinery etc., then raw material conversion rates could be defined as $\mathrm{mrR}_{\mathrm{r}} \mathrm{P}_{\mathrm{p}} \mathrm{F}_{\mathrm{f}}$. For this research, we assume that there is no significant difference or bill of materials for product $\mathrm{p}$ produced at each production facility. This constraint also assumes that raw materials received during the day will not be available for production until the next day.

Sum of production times used on day $d$ at production facility $f$ cannot exceed total available operating time (constraint $\mathrm{TPRODF}_{\mathrm{f}} \mathrm{D}_{\mathrm{d}}$ ). For each production facility and day,

$$
\sum_{p \in P F\{\}}\left(\left(\frac{1}{\text { unitperhr }}\right) * \operatorname{ProdP}_{\mathrm{p}} \mathrm{F}_{\mathrm{f}} \mathrm{D}_{\mathrm{d}}+\operatorname{sutimeF}_{\mathrm{f}} * \mathrm{SUF}_{\mathrm{f}} \mathrm{D}_{\mathrm{d}}+\operatorname{IdleF}_{\mathrm{f}} \mathrm{D}_{\mathrm{d}}\right)=8 * \text { maxshifts }
$$

$\mathrm{SUF}_{\mathrm{f}} \mathrm{D}_{\mathrm{d}}=[0,1]$. If setup times are negligible, these binary constraints may be relaxed.

Production of product $\mathrm{p}$ at production facility $\mathrm{f}$ on day $\mathrm{d}$ cannot occur unless the production facility is activated for production on that day (constraint $F S F_{\mathrm{f}} \mathrm{D}_{\mathrm{d}}$ ). For each production facility and day for each $p \in P F\{f\}$,

$$
\sum_{p \in P F\{\}} \operatorname{SUP}_{\mathrm{p}} \mathrm{F}_{\mathrm{f}} \mathrm{D}_{\mathrm{d}} \leq \mathrm{SUF}_{\mathrm{f}} \mathrm{D}_{\mathrm{d}}
$$

$\operatorname{SUP}_{\mathrm{p}} \mathrm{F}_{\mathrm{f}} \mathrm{D}_{\mathrm{d}}$ values attribute set up time to the production of individual product. If separate set up were required for each product, these equations would be replaced with sets of equations for set up of individual product. For this research, we assume that there is a single setup required if a production facility is to be activated for production during the day. $\mathrm{SUP}_{\mathrm{p}} \mathrm{F}_{\mathrm{f}} \mathrm{D}_{\mathrm{d}}$ in this formulation allocates production capacity to individual products. We, therefore, add a constraint that creates a single binary variable for each production facility during the day that accounts for setup to activate and shut down the production at production facility.

Raw materials inventory balance at production facility $\mathrm{f}$ (constraint $\mathrm{IBR}_{\mathrm{r}} \mathrm{F}_{\mathrm{f}} \mathrm{D}_{\mathrm{d}}$ ). For each production facility and day for each $r \in R F\{f\}$ and each $s \in S R\{r\}$,

$\operatorname{InvR}_{\mathrm{r}} \mathrm{F}_{\mathrm{f}} \mathrm{D}_{\mathrm{d}+1}=\operatorname{InvR}_{\mathrm{r}} \mathrm{F}_{\mathrm{f}} \mathrm{D}_{\mathrm{d}}-\sum_{\mathrm{p} \in \mathrm{PR}\{\mathrm{r}\}} \operatorname{mrR}_{\mathrm{r}} \mathrm{P}_{\mathrm{p}} * \operatorname{ProdP}_{\mathrm{p}} \mathrm{F}_{\mathrm{f}} \mathrm{D}_{\mathrm{d}}+\sum_{s \in \mathrm{SR}\{\mathrm{r}\}}\left(\operatorname{ShpR}_{\mathrm{r}} \mathrm{S}_{\mathrm{s}} \mathrm{F}_{\mathrm{f}} \mathrm{D}_{\mathrm{d}-\theta(\mathrm{s}, \mathrm{f})}+\operatorname{ItsR}_{\mathrm{r}} \mathrm{S}_{\mathrm{s}} \mathrm{F}_{\mathrm{f}} \mathrm{D}_{\mathrm{d}}\right)$

Note that the ItsR $R_{r} S_{s} F_{f} D_{d}$ variables are defined only for $(r, s, f, d)$ combinations where there are raw materials in transit at beginning of the planning horizon and are scheduled to arrive at production facility $\mathrm{f}$ on day d for each $d \in D R M S\{r, s, f\}$.

Place order of raw material $\mathrm{r}$ at beginning of day $\mathrm{d}$ to ensure safety stock at production facility $\mathrm{f}$ (constraint $\mathrm{MNOR}_{\mathrm{r}} \mathrm{F}_{\mathrm{f}} \mathrm{D}_{\mathrm{d}}$ ). For each production facility and day for each $r \in R F\{f\}$ and each $s \in S R\{r\}$,

$\sum_{s \in S R\{r\}}\left(O R_{r} S_{s} F_{f} D_{d}+0 O R_{r} S_{s} F_{f} D_{d}\right)+\operatorname{InvR}_{r} F_{f} D_{d} \geq \operatorname{mininvR} R_{r} F_{f}-U_{S R} F_{f} D_{d-1}$.

Note that under storage of raw materials could occur at the beginning of Day 1. 
Restrict order of raw material $\mathrm{r}$ at beginning of day $\mathrm{d}$ to prevent overstock at production facility $\mathrm{f}$ (constraint $\mathrm{MXOR}_{\mathrm{r}} \mathrm{F}_{\mathrm{f}} \mathrm{D}_{\mathrm{d}}$ ). For each production facility and day for each $r \in R F\{f\}$ and each $s \in S R\{r\}$,

$\sum_{s \in S R\{r\}}\left(O R_{r} S_{s} F_{f} D_{d}+0 O R_{r} S_{s} F_{f} D_{d}\right)+I n v R_{r} F_{f} D_{d} \leq \operatorname{maxinvR}_{r} F_{f}+0 S R_{r} F_{f} D_{d-1}$

Note that over storage of raw materials could occur at the beginning of Day 1.

Update under storage (constraint $\mathrm{AUSR}_{\mathrm{r}} \mathrm{F}_{\mathrm{f}} \mathrm{D}_{\mathrm{d}}$ ) and overstocking (constraint $\mathrm{AOSR}_{\mathrm{r}} \mathrm{F}_{\mathrm{f}} \mathrm{D}_{\mathrm{d}}$ ) of raw material $\mathrm{r}$ at production facility $\mathrm{f}$ at the end of day $\mathrm{d}$. For each production facility and day for each $r \in R F\{f\}$ and each $s \in S R\{r\}$,

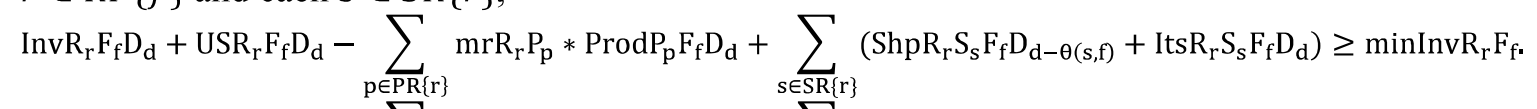

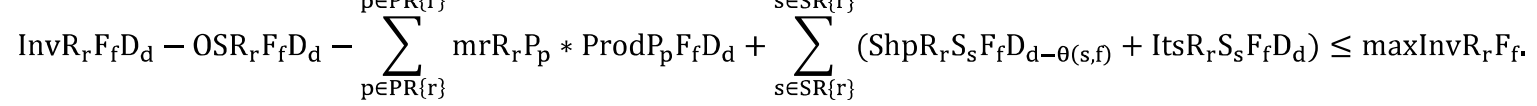

Note that the ItsR $\mathrm{S}_{\mathrm{s}} \mathrm{F}_{\mathrm{f}} \mathrm{D}_{\mathrm{d}}$ variables are defined only for $(\mathrm{r}, \mathrm{s}, \mathrm{f}, \mathrm{d})$ combinations where there are raw materials in transit at beginning of the planning horizon and are scheduled to arrive at production facility $\mathrm{f}$ on day $\mathrm{d}$ for each $d \in D R M S\{r, s, f\}$.

Total units of raw material $r$ shipped from supplier $s$ at the end of day $d$ to satisfy orders acknowledged from production facility $f$ at beginning of that day (constraint $\mathrm{DLVR}_{\mathrm{r}} \mathrm{S}_{\mathrm{s}} \mathrm{F}_{\mathrm{f}} \mathrm{D}_{\mathrm{d}}$ ). For each day and each $s \in \mathrm{SR}\{r\}$ for each production facility,

$\operatorname{ShpR}_{\mathrm{r}} \mathrm{S}_{\mathrm{s}} \mathrm{F}_{\mathrm{f}} \mathrm{D}_{\mathrm{d}} \geq 0 \mathrm{R}_{\mathrm{r}} \mathrm{S}_{\mathrm{s}} \mathrm{F}_{\mathrm{f}} \mathrm{D}_{\mathrm{d}}$.

Update outstanding orders of raw material $\mathrm{r}$ at production facility $\mathrm{f}$ at beginning of day $\mathrm{d}$ (constraint OOUR $\mathrm{S}_{\mathrm{r}} \mathrm{F}_{\mathrm{f}} \mathrm{D}_{\mathrm{d}}$ ). For each production facility and day for each $r \in \operatorname{RF}\{f\}$ and each $d \in D R M S\{r, s, f\}$,

$00 R_{r} S_{s} F_{f} D_{d+1}=00 R_{r} S_{s} F_{f} D_{d}+0 R_{r} S_{s} F_{f} D_{d}-S h p R_{r} S_{s} F_{f} D_{d-\theta(s, f)}-I_{t s R} R_{r} S_{s} F_{f} D_{d}$.

Note that the ItsR $\mathrm{S}_{\mathrm{r}} \mathrm{F}_{\mathrm{f}} \mathrm{D}_{\mathrm{d}}$ variables are defined only for $(r, s, f, d)$ combinations where there are raw materials in transit at beginning of the planning horizon and are scheduled to arrive at production facility $\mathrm{f}$ on day $\mathrm{d}$ for each $d \in D R M S\{r, s, f\}$. OOR $\mathrm{S}_{\mathrm{r}} \mathrm{F}_{\mathrm{f}} \mathrm{D}_{1}$ should include sum of the $\operatorname{ItsR}_{\mathrm{r}} \mathrm{S}_{\mathrm{S}} \mathrm{F}_{\mathrm{F}} \mathrm{D}_{\mathrm{d}}$ values for each day with scheduled arrivals.

Update raw materials in transit from supplier $s$ to production facility $f$ (constraint $\mathrm{RITR}_{\mathrm{r}} \mathrm{S}_{\mathrm{s}} \mathrm{F}_{\mathrm{f}} \mathrm{D}_{\mathrm{d}}$ ) at beginning of day $\mathrm{d}$. For each production facility and day for each $r \in \operatorname{RF}\{f\}$ and each $d \in$ $D R M S\{r, s, f\}$,

$\operatorname{TrR}_{r} S_{s} F_{f} D_{d+1}=\operatorname{TrR}_{r} S_{s} F_{f} D_{d}+\operatorname{ShpR}_{r} S_{s} F_{f} D_{d}-\operatorname{ShpR}_{r} S_{s} F_{f} D_{d-\theta(s, f)}-I_{t s R} S_{s} F_{f} D_{d}$

Note that the ItsR $\mathrm{S}_{\mathrm{r}} \mathrm{S}_{\mathrm{f}} \mathrm{D}_{\mathrm{d}}$ variables are defined only for (r, s, f, d) combinations where there are raw materials in transit at beginning of the planning horizon and are scheduled to arrive at production facility $\mathrm{f}$ on day $\mathrm{d}$ for each $d \in D R M S\{r, s, f\}$. $\operatorname{TrRrSsFFD}_{1}$ is set to sum of the $\operatorname{ItsR}_{\mathrm{r}} \mathrm{S}_{\mathrm{s}} \mathrm{F}_{\mathrm{f}} \mathrm{D}_{\mathrm{d}}$ values for each day with scheduled arrivals of raw materials.

Place order for product $\mathrm{p}$ at the beginning of day $\mathrm{d}$ to ensure desired safety stock at warehouse $\mathrm{w}$ (constraint $\mathrm{MNOP}_{\mathrm{p}} \mathrm{W}_{\mathrm{w}} \mathrm{D}_{\mathrm{d}}$ ). For each warehouse and day for each $p \in P W\{w\}$,

$\sum_{f \in F\{f\}}\left(O_{p} F_{f} W_{w} D_{d}+00 P_{p} F_{f} W_{w} D_{d}\right)+\operatorname{InvP}_{p} W_{w} D_{d} \geq \operatorname{mininvP}_{p} W_{w}-U_{\text {SP }} W_{w} D_{d-1}$

Note that under storage of products can occur with associated penalty.

Restrict order of product $\mathrm{p}$ at the beginning of day $\mathrm{d}$ to prevent overstock at warehouse $\mathrm{w}$ (constraint $\left.\mathrm{MXOP}_{\mathrm{p}} \mathrm{W}_{\mathrm{w}} \mathrm{D}_{\mathrm{d}}\right)$. For each warehouse and day for each $p \in P W\{w\}$,

$\sum_{f \in F\{f\}}\left(O P_{p} F_{f} W_{w} D_{d}+00 P_{p} F_{f} W_{w} D_{d}\right)+\operatorname{InvP}_{p} W_{w} D_{d} \leq \operatorname{maxinv} P_{p} W_{w}+O \operatorname{OSP}_{p} W_{w} D_{d-1}$. 
Note that over storage of products can occur with associated penalty.

Produce sufficient product $\mathrm{p}$ across plants to cover orders and provide production system-wide safety stocks (constraint MNSYSP $\mathrm{P}_{\mathrm{p}}$ ). For each day for each product across all plants,

$\sum_{f \in F\{f\}}\left(\operatorname{Prod} P_{p} F_{f} D_{d}+\operatorname{InvP}_{p} F_{f} D_{d}\right) \geq \sum_{f \in F\{f\}} \sum_{w \in P W\{w\}} O P_{p} F_{f} W_{w} D_{d}+\operatorname{minsysinv} P_{p}$.

Restrict production of product $\mathrm{p}$ across plants on day $\mathrm{d}$ to prevent overstock in the production system (constraint MXSYSP ${ }_{\mathrm{p}} \mathrm{D}_{\mathrm{d}}$ ). For each day for each product across all plants,

$\sum_{f \in F\{f\}}\left(\operatorname{Prod} P_{p} F_{f} D_{d}+\operatorname{InvP}_{p} F_{f} D_{d}\right) \leq \sum_{f \in F\{f\}} \sum_{w \in P W\{w\}} O P_{p} F_{f} W_{w} D_{d}+\operatorname{maxsysinv} P_{p}$.

Ship sufficient finished goods from production facility $\mathrm{f}$ to cover orders placed at warehouse $\mathrm{w}$ on day $d$ (constraint $\operatorname{DLVP}_{\mathrm{p}} \mathrm{F}_{\mathrm{f}} \mathrm{W}_{\mathrm{w}} \mathrm{D}_{\mathrm{d}}$ ). For each production facility and day for each $\mathrm{p} \in \mathrm{PF}\{\mathrm{f}\}$ and each $p \in P W\{w\}$,

$\operatorname{ShpP}_{\mathrm{p}} \mathrm{F}_{\mathrm{f}} \mathrm{W}_{\mathrm{w}} \mathrm{D}_{\mathrm{d}} \geq O \mathrm{OP}_{\mathrm{p}} \mathrm{F}_{\mathrm{f}} \mathrm{W}_{\mathrm{w}} \mathrm{D}_{\mathrm{d}}$.

Update over storage (constraint $\operatorname{AOSP}_{\mathrm{p}} \mathrm{F}_{\mathrm{f}} \mathrm{D}_{\mathrm{d}}$ ) and under storage (constraint $\mathrm{AUSP}_{\mathrm{p}} \mathrm{F}_{\mathrm{f}} \mathrm{D}_{\mathrm{d}}$ ) of product $\mathrm{p}$ at production facility $\mathrm{f}$ at the end of day $\mathrm{d}$. For each production facility and day for each $p \in P F\{f\}$ and each $p \in P W\{w\}$,

$\operatorname{InvP}_{p} F_{f} D_{d}-\operatorname{OSP}_{p} F_{f} D_{d}+\operatorname{ProdP}_{p} F_{f} D_{d}-\sum_{w \in W P\{p\}}\left(\operatorname{ShpP}_{p} F_{f} W_{w} D_{d}+\operatorname{AltP}_{p} F_{f} W_{w} D_{d}\right) \leq \operatorname{maxInvP} F_{p} F_{f}$.
$\operatorname{InvP}_{p} F_{f} D_{d}+\operatorname{USP}_{p} F_{f} D_{d}+\operatorname{ProdP}_{p} F_{f} D_{d}-\sum_{w \in W P\{p\}}\left(\operatorname{ShpP}_{p} F_{f} W_{w} D_{d}+\operatorname{AltP}_{p} F_{f} W_{w} D_{d}\right) \geq \operatorname{minInvP} F_{p} F_{f}$.

Limit shipments of product $p$ from production facility $f$ to warehouses on day $d$ to the amount available in production facility inventory (constraint $\mathrm{SLP}_{\mathrm{p}} \mathrm{F}_{\mathrm{F}} \mathrm{D}_{\mathrm{d}}$ ). For each production facility and day for each $p \in P F\{f\}$ and each $p \in P W\{w\}$,

$\sum_{w \in W P\{p\}}\left(\operatorname{ShpP}_{\mathrm{p}} \mathrm{F}_{\mathrm{f}} \mathrm{W}_{\mathrm{w}} \mathrm{D}_{\mathrm{d}}+\operatorname{AltP}_{\mathrm{p}} \mathrm{F}_{\mathrm{f}} \mathrm{W}_{\mathrm{w}} \mathrm{D}_{\mathrm{d}}\right) \leq \operatorname{InvP}_{\mathrm{p}} \mathrm{F}_{\mathrm{f}} \mathrm{D}_{\mathrm{d}}$

This also implies that production of product $p$ during day $d$ will not be available for delivery until the next day.

Account for inventory balance of products at production facility $\mathrm{f}$ at the end of day $\mathrm{d}$ (constraint $\left.\mathrm{IBP}_{\mathrm{p}} \mathrm{F}_{\mathrm{f}} \mathrm{D}_{\mathrm{d}}\right)$. For each production facility and day for each $p \in P F\{f\}$ and each $p \in P W\{w\}$,

$\operatorname{InvP}_{\mathrm{p}} \mathrm{F}_{\mathrm{f}} \mathrm{D}_{\mathrm{d}+1}=\operatorname{InvP}_{\mathrm{p}} \mathrm{F}_{\mathrm{f}} \mathrm{D}_{\mathrm{d}}+\operatorname{ProdP}_{\mathrm{p}} \mathrm{F}_{\mathrm{f}} \mathrm{D}_{\mathrm{d}}-\sum_{\mathrm{w} \in \mathrm{WP}\{\mathrm{p}\}}\left(\operatorname{ShpP}_{\mathrm{p}} \mathrm{F}_{\mathrm{f}} \mathrm{W}_{\mathrm{w}} \mathrm{D}_{\mathrm{d}}+\operatorname{AltP}_{\mathrm{p}} \mathrm{F}_{\mathrm{f}} \mathrm{W}_{\mathrm{w}} \mathrm{D}_{\mathrm{d}}\right)$.

Deliver goods from warehouse or alternative source (production facility) to satisfy customer demand and acknowledge lost sales if inventory is insufficient (constraint $\operatorname{DLVP}_{\mathrm{p}} \mathrm{W}_{\mathrm{w}} \mathrm{D}_{\mathrm{d}}$ ). For each warehouse and day for each $p \in P W\{w\}$,

$\operatorname{DelP}_{\mathrm{p}} \mathrm{W}_{\mathrm{w}} \mathrm{D}_{\mathrm{d}}+\mathrm{LSP}_{\mathrm{p}} \mathrm{W}_{\mathrm{w}} \mathrm{D}_{\mathrm{d}}+\sum_{\mathrm{f} \in \mathrm{F}\{\mathrm{f}\}} \operatorname{AltP}_{\mathrm{p}} \mathrm{F}_{\mathrm{f}} \mathrm{W}_{\mathrm{w}} \mathrm{D}_{\mathrm{d}}=\operatorname{DemP}_{\mathrm{p}} \mathrm{W}_{\mathrm{w}} \mathrm{D}_{\mathrm{d}}$

Account for inventory balance of product $\mathrm{p}$ at warehouse $\mathrm{w}$ recognizing inbound shipping delays (constraint $\mathrm{IBP}_{\mathrm{p}} \mathrm{W}_{\mathrm{w}} \mathrm{D}_{\mathrm{d}}$ ) at the end of day d. For each warehouse and day for each $p \in P W\{w\}$,

$\operatorname{InvP}_{p} W_{w} D_{d+1}=\operatorname{InvP}_{p} W_{w} D_{d}-\operatorname{DelP}_{p} W_{w} D_{d}+\sum_{f \in F\{f\}}\left(\operatorname{ShpP}_{p} F_{f} W_{w} D_{d-\delta(f, w)}+\operatorname{ItsP}_{p} F_{f} W_{w} D_{d}\right)$. 
Note that the Its $\mathrm{P}_{\mathrm{p}} \mathrm{F}_{\mathrm{F}} \mathrm{W}_{\mathrm{W}} \mathrm{D}_{\mathrm{d}}$ variables are defined only for $(\mathrm{p}, \mathrm{f}, \mathrm{w}, \mathrm{d})$ combinations where there are finished goods in transit at the beginning of the planning horizon and are scheduled to arrive at warehouse w on day $\mathrm{d}$ for each $d \in \operatorname{DFGS}\{p, f, w\}$.

Update over storage (constraint $\operatorname{AOSP}_{\mathrm{p}} \mathrm{W}_{\mathrm{w}} \mathrm{D}_{\mathrm{d}}$ ) or under storage (constraint $\mathrm{AUSP}_{\mathrm{p}} \mathrm{W}_{\mathrm{w}} \mathrm{D}_{\mathrm{d}}$ ) of product $\mathrm{p}$ at warehouse $w$ at the end of day $d$. For each warehouse and day for each $p \in P W\{w\}$,

$\operatorname{InvP}_{\mathrm{p}} \mathrm{W}_{\mathrm{w}} \mathrm{D}_{\mathrm{d}}+\mathrm{USP}_{\mathrm{p}} \mathrm{W}_{\mathrm{w}} \mathrm{D}_{\mathrm{d}}-\operatorname{DelP}_{\mathrm{p}} \mathrm{W}_{\mathrm{w}} \mathrm{D}_{\mathrm{d}}+\sum_{\mathrm{f} \in \mathrm{F}\{\mathrm{f}\}}\left(\operatorname{ShpP}_{\mathrm{p}} \mathrm{F}_{\mathrm{f}} \mathrm{W}_{\mathrm{w}} \mathrm{D}_{\mathrm{d}-\delta(\mathrm{f}, \mathrm{w})}+\operatorname{ItsP}_{\mathrm{p}} \mathrm{F}_{\mathrm{f}} \mathrm{W}_{\mathrm{w}} \mathrm{D}_{\mathrm{d}}\right) \geq \operatorname{mininvP}_{\mathrm{p}} \mathrm{W}_{\mathrm{w}}$.
$\operatorname{InvP}_{\mathrm{p}} \mathrm{W}_{\mathrm{w}} \mathrm{D}_{\mathrm{d}}-\operatorname{OSP}_{\mathrm{p}} \mathrm{W}_{\mathrm{w}} \mathrm{D}_{\mathrm{d}}-\operatorname{DelP}_{\mathrm{p}} \mathrm{W}_{\mathrm{w}} \mathrm{D}_{\mathrm{d}}+\sum_{\mathrm{f} \in \mathrm{F}\{f\}}\left(\operatorname{ShpP}_{\mathrm{p}} \mathrm{F}_{\mathrm{f}} \mathrm{W}_{\mathrm{w}} \mathrm{D}_{\mathrm{d}-\delta(\mathrm{f}, \mathrm{w})}+\operatorname{ItsP}_{\mathrm{p}} \mathrm{F}_{\mathrm{f}} \mathrm{W}_{\mathrm{w}} \mathrm{D}_{\mathrm{d}}\right) \leq \operatorname{maxinvP}_{\mathrm{p}} \mathrm{W}_{\mathrm{w}}$.

Note that the Its $\mathrm{P}_{\mathrm{p}} \mathrm{F}_{\mathrm{F}} \mathrm{W}_{\mathrm{W}} \mathrm{D}_{\mathrm{d}}$ variables are defined only for $(\mathrm{p}, \mathrm{f}, \mathrm{w}, \mathrm{d})$ combinations where there are finished goods in transit at the beginning of the planning horizon and are scheduled to arrive at warehouse w on day d for each $d \in D F G S\{p, f, w\}$.

Update outstanding orders for product $\mathrm{p}$ at warehouse $\mathrm{w}$ at the end of day $\mathrm{d}$ (constraint OOUP $\mathrm{p}_{\mathrm{p}} \mathrm{F}_{\mathrm{F}} \mathrm{W}_{\mathrm{w}} \mathrm{D}_{\mathrm{d}}$ ). For each warehouse and day for each $p \in P W\{w\}$ and each $d \in \operatorname{DFGS}\{p, f, w\}$, $0 O P_{p} F_{f} W_{w} D_{d+1}=0 O P_{p} F_{f} W_{w} D_{d}+0 P_{p} F_{f} W_{w} D_{d}-S h p P_{p} F_{f} W_{w} D_{d-\delta(f, w)}-\operatorname{ItsP}_{p} F_{f} W_{w} D_{d}$.

Note that the Its $\mathrm{P}_{\mathrm{p}} \mathrm{F}_{\mathrm{F}} \mathrm{W}_{\mathrm{W}} \mathrm{D}_{\mathrm{d}}$ variables are defined only for $(\mathrm{p}, \mathrm{f}, \mathrm{w}, \mathrm{d})$ combinations where there are finished goods in transit at the beginning of the planning horizon and are scheduled to arrive at warehouse $\mathrm{w}$ on day $\mathrm{d}$ for each $d \in D F G S\{p, f, w\}$. OOP $\mathrm{F}_{\mathrm{F}} \mathrm{W}_{\mathrm{w}} \mathrm{D}_{1}$ should include sum of the $\mathrm{Its}_{\mathrm{p}} \mathrm{F}_{\mathrm{F}} \mathrm{W}_{\mathrm{W}} \mathrm{D}_{\mathrm{d}}$ values for each day with scheduled arrivals.

Update finished goods in transit to reflect shipments and receipts (constraint $\mathrm{GITP}_{\mathrm{p}} \mathrm{F}_{\mathrm{f}} \mathrm{W}_{\mathrm{w}} \mathrm{D}_{\mathrm{d}}$ ) at the end of day d. For each warehouse and day for each $p \in P W\{w\}$ and each $d \in D F G S\{p, f, w\}$, $\operatorname{TrP}_{\mathrm{p}} \mathrm{F}_{\mathrm{f}} \mathrm{W}_{\mathrm{w}} \mathrm{D}_{\mathrm{d}+1}=\operatorname{TrP}_{\mathrm{p}} \mathrm{F}_{\mathrm{f}} \mathrm{W}_{\mathrm{w}} \mathrm{D}_{\mathrm{d}}+\operatorname{ShP}_{\mathrm{p}} \mathrm{F}_{\mathrm{f}} \mathrm{W}_{\mathrm{w}} \mathrm{D}_{\mathrm{d}}-\operatorname{ShpP}_{\mathrm{p}} \mathrm{F}_{\mathrm{f}} \mathrm{W}_{\mathrm{w}} \mathrm{D}_{\mathrm{d}-\delta(\mathrm{f}, \mathrm{w})}-\operatorname{ItsP}_{\mathrm{p}} \mathrm{F}_{\mathrm{f}} \mathrm{W}_{\mathrm{w}} \mathrm{D}_{\mathrm{d}}$.

Note that the Its $\mathrm{P}_{\mathrm{p}} \mathrm{F}_{\mathrm{F}} \mathrm{W}_{\mathrm{W}} \mathrm{D}_{\mathrm{d}}$ variables are defined only for $(\mathrm{p}, \mathrm{f}, \mathrm{w}, \mathrm{d})$ combinations where there are finished goods in transit at the beginning of the planning horizon and are scheduled to arrive at warehouse w on day $\mathrm{d}$ for each $d \in D F G S\{p, f, w\}$. $\operatorname{Tr}_{\mathrm{p}} \mathrm{F}_{\mathrm{f}} \mathrm{W}_{\mathrm{w}} \mathrm{D}_{1}$ is set to sum of the $\operatorname{Its}_{\mathrm{p}} \mathrm{F}_{\mathrm{f}} \mathrm{W}_{\mathrm{w}} \mathrm{D}_{\mathrm{d}}$ values for each day with scheduled arrivals.

As formulated with the warehouse inventory balance constraint (23), products that arrive in a day may be cross-docked and shipped out immediately if there is demand for them on that day rather than putting them into inventory. Such shipments could be delayed until the next day by adding a constraint (constraint $\mathrm{CDP}_{\mathrm{p}} \mathrm{W}_{\mathrm{W}} \mathrm{D}_{\mathrm{d}}$ ) that delivery of product $\mathrm{p}$ at warehouse $\mathrm{w}$ in a day can't exceed the beginning inventory of that product in that day. For each warehouse and day for each $p \in P W\{w\}$,

$\operatorname{DelP}_{\mathrm{p}} \mathrm{W}_{\mathrm{w}} \mathrm{D}_{\mathrm{d}} \leq \operatorname{InvP}_{\mathrm{p}} \mathrm{W}_{\mathrm{w}} \mathrm{D}_{\mathrm{d}}$.

All variables are nonnegative. To facilitate extraction of the solution in the report generator, we define variable $A R R P_{p} F_{f} W_{W} D_{d}$ to be the finished goods shipped from all production facilities that arrive at the warehouse in day $\mathrm{d}$ which will be shipped in this planning horizon and establish their equality in constraints that define inbound freight (constraint IBP $\mathrm{p}_{\mathrm{f}} \mathrm{F}_{\mathrm{W}} \mathrm{W}_{\mathrm{d}}$ ). We also define variable $\mathrm{ARRR}_{\mathrm{r}} \mathrm{S}_{\mathrm{s}} \mathrm{F}_{\mathrm{f}} \mathrm{D}_{\mathrm{d}}$ to be the amount of raw material $r$ shipped from supplier $s$ to arrive at production facility $f$ on day $d$. They are set equal to the corresponding outbound shipments as follows (constraint $I_{B} R_{r} S_{s} F_{f} D_{d}$ ),

$\operatorname{ARRP}_{\mathrm{p}} \mathrm{F}_{\mathrm{f}} \mathrm{W}_{\mathrm{w}} \mathrm{D}_{\mathrm{d}}=\operatorname{ShpP}_{\mathrm{p}} \mathrm{F}_{\mathrm{f}} \mathrm{W}_{\mathrm{w}} \mathrm{D}_{\mathrm{d}-\delta(f, \mathrm{w})}$.

$\operatorname{ARRR}_{\mathrm{r}} \mathrm{S}_{\mathrm{s}} \mathrm{F}_{\mathrm{f}} \mathrm{D}_{\mathrm{d}}=\operatorname{ShpR}_{\mathrm{r}} \mathrm{S}_{\mathrm{s}} \mathrm{F}_{\mathrm{f}} \mathrm{D}_{\mathrm{d}-\theta(\mathrm{s}, \mathrm{f})}$.

Note that variables of $\operatorname{Its}_{\mathrm{p}} \mathrm{F}_{\mathrm{f}} \mathrm{W}_{\mathrm{W}} \mathrm{D}_{\mathrm{d}}$ and ItsRrSsFfDd represent goods in transit to arrive as a result of initial conditions, while that of $\operatorname{ShpPpFfW}{ }_{\mathrm{W}} \mathrm{Dd}$ and $\mathrm{ShpR}_{\mathrm{r}} \mathrm{S}_{\mathrm{S}} \mathrm{F}_{\mathrm{f}} \mathrm{D}_{\mathrm{d}}$ indicate when goods arrive from shipments in the current planning frame. 